\title{
Los grupos multimedia en España ante el reto de la convergencia de medios (2012-2013) ${ }^{1}$
}

\section{Media groups in Spain in the challenge of media convergence (2012-2013)}

\author{
José María Legorburu Hortelano. Universidad CEU San Pablo \\ María Sánchez Martínez. Universidad CEU San Pablo
}

Recibido: 25-III-2014 - Aceptado: 6-X-2014

Resumen:

Este artículo de investigación persigue confirmar si en España confluyen las condiciones necesarias para la convergencia de medios, además de describir y evaluar el grado de desarrollo de las diferentes estrategias que desarrollan los grupos multimedia al respecto. Así, la investigación se fundamentó en una metodología cualitativa basada en la realización de entrevistas en profundidad bajo cuestionario a una muestra de responsables de estos grupos. Los resultados desvelan que, aunque la convergencia es una realidad, su desarrollo es incipiente. Si bien las corporaciones son conscientes de su importancia, no la consideran una herramienta eficaz para renovar y adaptar sus modelos de negocio al nuevo escenario impuesto por la web y las tecnologías emergentes.

Palabras clave:

Convergencia de medios, Grupos multimedia, España, Tecnologías emergentes, Modelos de negocio.

Abstract:

The following research has the aim to confirm whether the conditions for media convergence in Spain had been successfully completed in addition to describing and assessing the degree of development of the different strategies applied by the multimedia groups. Thus, the research was based on a qualitative methodology developing in-depth interviews with a questionnaire with several managers of the sample groups. The findings suggest that although convergence is a reality, its development is still in its early stages. Whilst corporations are aware of its importance, they do not take it into consideration as an effective tool to renew and adapt their business models to the new circumstances imposed by the Web and the emerging technologies.

Key words:

Media Convergence, Media Groups; Spain, Emerging Technologies, Business Models.

1 El presente artículo es fruto del Proyecto de Investigación titulado "Estudio de los modelos de convergencia de medios en España" (USBS-USP-BS-PPC10/2011), desarrollado por el Grupo de Investigación en Convergencia de Medios INCIRTV de la Universidad CEU San Pablo (Madrid, España) en el periodo 2011-2013, evaluado positivamente por la Agencia Nacional de Evaluación y Prospectiva (ANEP) y financiado por el Banco Santander. 


\section{Introducción}

A pesar de que hoy en día, la convergencia de medios sigue estando de plena actualidad, conviene recordar que no se trata de un concepto nuevo, puesto que tiene su origen en la década de los años 70 del pasado siglo XX y distintos autores lo han venido desarrollando desde entonces. De Sola Pool (1983) fue el pionero en hacer referencia académica al respecto de este fenómeno en su obra Technologies of Freedom, motivo por el cual Jenkins le denomina "el profeta de la convergencia". Años después, en 1995, Negroponte profundizaba en la influencia que habría de tener el paso de los sistemas analógicos a los digitales, no ya en los contenidos de los medios de comunicación, sino en la propia sociedad y Fidler (1997) aportaba el concepto de mediamorfosis.

Posteriormente, en esta misma línea, Pavlik $(1993,2004)$, atento a la transformación de las empresas de este sector, anunció la desaparición de las fronteras entre los diferentes medios y la multiplicación de sus posibilidades de difusión de contenidos. Recién iniciado el siglo XXI, Castells Oliván (2001) apuntaba a internet como la clave para el progreso con su concepto de "sociedad en red" o "Galaxia Internet", parafraseando a McLuhan. Por su lado, Jenkins $(2004,2006)$ profundizaba en la influencia de la convergencia en el cambio cultural acuñando el término transmedia.

Asimismo, Flynn (2000) describió la convergencia como un proceso sistémico, sugiriendo tres áreas de convergencia basadas en los aparatos, las redes y los contenidos y abriendo una corriente en la que han trabajado otros investigadores. Son relevantes en este sentido las aportaciones de Dailey, Demo y Spillmann (2003), que proporcionan un marco para la comprensión de la convergencia a través de la identificación de cinco niveles de actividad entre las organizaciones (promoción cruzada, clonación, cooperación competitiva, intercambio de contenidos y convergencia completa); Gordon (2003), que agrupa las convergencias empresarial y organizacional en una sola, a la que denomina "convergencia estructural"; Salaverría Aliaga (2003), que distingue hasta cuatro dimensiones de la convergencia (empresarial, tecnológica, profesional y comunicativa) o de Dennis (2003), que propone cinco posibles áreas de convergencia: tecnológica, regulatoria, empresarial, de contenido y de recursos humanos.

Así, la convergencia se ha ido configurando como un concepto extraordinariamente complejo, polisémico y multifacético -también controvertido y discutible-, que en el campo de la comunicación hace referencia a numerosos procesos de distinta índole: empresarial, tecnológica, organizativa, profesional y de producción de contenidos. Salaverría Aliaga, García Avilés y Masip Masip (2008) la definen en estos términos:

“La convergencia periodística es un proceso multidimensional que, propiciado por la implantación generalizada de las tecnologías digitales, afecta actualmente a las empresas de medios de comunicación y conforme al cual las esferas tecnológica, empresarial, profesional y de contenidos de esas empresas experimentan respectivos fenómenos de mudanza, caracterizados por una integración de herramientas, espacios, métodos de trabajo y lenguajes anteriormente disgregados". 
De esta forma, lo que inicialmente constituía una mera conjetura, se plasma en la actualidad en modelos concretos de aplicación dentro y fuera de nuestras fronteras que, en buena medida, resultan imprescindibles para el sostenimiento de las empresas de comunicación en un mercado marcado por la sobreabundancia de oferta, especialmente en el ámbito de internet y del audiovisual -sobre todo a raíz de la implantación de la Televisión Digital Terrestre (TDT) y de la Web TV, así como de la expansión de los dispositivos móviles- y por la prolongada crisis económica, que ha reducido drásticamente de los ingresos por comercialización publicitaria.

En este sentido, se puede afirmar que el sector de los medios de comunicación se encuentra desde hace aproximadamente un lustro ante una encrucijada (Diezhandino Nieto, 2012). Mientras se beneficia de los avances tecnológicos en los más diversos procedimientos: gestión, producción y distribución; se ve abocado a efectuar-a regañadientes- profundos cambios en sus estructuras, sin saber a ciencia cierta cuál será su alcance, a la vez que busca precipitadamente un nuevo modelo de negocio que permita su supervivencia.

Una vez confirmado que, efectivamente, se dan las condiciones necesarias para que se desarrolle la convergencia de medios en España, es decir, que se han producido los avances tecnológicos necesarios (digitalización), ha tenido lugar la indispensable concentración empresarial, dando lugar a los grupos multimedia, y se ha alcanzado la imprescindible polivalencia profesional, el presente trabajo tiene como objetivo de investigación describir y evaluar las diferentes estrategias convergentes que en los últimos tiempos vienen desarrollando los grupos multimedia españoles.

En este mismo sentido, la finalidad de este estudio es no solamente contribuir al avance del conocimiento en el ámbito de la comunidad científica sino que, a la vez, pretende ser de utilidad en el perfeccionamiento de las diferentes estrategias convergentes que vienen empleado estas corporaciones: consolidar o mejorar su imagen de marca, ampliar su difusión y, por lo tanto, su audiencia, con el consiguiente incremento de los ingresos por comercialización publicitaria y, en último término, gestionar de una forma más eficiente los recursos que destinan a la generación de contenidos, con el correspondiente ahorro de costes.

A continuación, se describen, en primer lugar, el escenario actual de la convergencia de medios en España, fruto de la primera fase del estudio y, seguidamente, los resultados más relevantes obtenidos de la investigación realizada mediante los cuestionarios y el análisis comparativo.

\section{El escenario actual de la convergencia de medios en España}

Resulta evidente que la convergencia de medios solamente es factible si, de forma previa, se han dado las condiciones necesarias; es decir, si se han desarrollado las tecnologías necesarias -en este caso, digitales-y se han aplicado en los medios de comunicación; si han surgido grupos multimedia con entidad suficiente fruto de procesos de concentración empresa- 
rial y, por último, si los profesionales de la información han ido ganando en polivalencia, tanto en lo que se refiere a la realización de varias tareas informativas de forma simultánea, como en su capacidad para adaptar su trabajo en función de los distintos medios a través de los cuales se va a difundir. Todas ellas posibilitan la convergencia de los contenidos y su multidifusión.

Solamente a partir de este momento, sería posible desarrollar la convergencia en un medio de comunicación, si bien es cierto que puede darse la paradoja -y de hecho, ocurre- de que, a pesar de cumplirse todas las condiciones en un determinado medio para sacar provecho de su aplicación, no se emplee -por falta de liderazgo en su puesta en marcha o por la natural resistencia al cambio de las estructuras y los profesionales- o de que, por el contrario, se esté echando mano de ella sin una conciencia clara de que se está usando, lo que da lugar a múltiples ineficiencias.

En todo caso, en lo que se refiere a España y como pone de manifiesto la primera parte de la presente investigación, se puede afirmar que hace tiempo que se cumplen todas estas condiciones.

\subsection{El imparable avance de la digitalización}

En lo que se refiere al punto de vista tecnológico, la digitalización y el uso de internet han venido implantándose paulatinamente-aunque de forma asíncrona- en los diferentes medios de comunicación tradicionales: prensa, radio y televisión. Según explican González Díez y Pérez Cuadrado (2013: 25-74), la prensa ha evolucionado vertiginosamente en un plazo de apenas medio siglo. Tras 200 años de tipografía y composición en caliente se pasó a la fotocomposición y el offset en la década de los 60 del siglo pasado y a la incorporación de los primeros ordenadores diez años después. La autoedición y el PostScript hicieron el resto a finales de los 80 , eliminando la frontera entre los talleres y la redacción y reduciendo de forma drástica el personal técnico, puesto que buena parte de sus tareas fueron asumidas por los periodistas.

A pesar de que las empresas no confiaban en el éxito y seguían combinando el uso de las nuevas tecnologías con los procedimientos tradicionales, este se produjo. El primer periódico totalmente digital, ya no de España sino del mundo, fue el español El Sol (1990), con tecnología Macintosh de Apple. A partir de ahí, la digitalización se extendió como una mancha de aceite por el sector y abrió la puerta a la prensa gratuita. Más recientemente, los procesos de producción se han ido perfeccionando con sistemas más eficientes y los de distribución con el empleo del PDF.

La irrupción de la prensa española en internet se produjo, según relata García Avilés (2013: 183-230) en noviembre de 1994 con las ediciones de El Periódico de Catalunya y El Mundo. El primer periódico en contar con una versión en internet fue Avui en abril de 1995. La Estrella Digital, surgido en junio de 1998, es considerado el primer cibermedio generalista nativo español, que abrió la puerta a la aparición y consolidación de otros medios exclusivos de la Red, basados sobre todo en la opinión y la información confidencial. Los periódicos tradicionales han potenciado sus contenidos en la web con redise- 
ños y mejoras constantes, desechando la idea inicial de repetir los patrones de sus ediciones impresas y abriéndose a los contenidos audiovisuales y a la participación de los usuarios. También se ponían en marcha en 2010 y 2011, respectivamente, los quioscos digitales: Orbyt y Kiosko y más.

Como en el resto del mundo, la prensa atraviesa por un momento de gran incertidumbre a la búsqueda de un nuevo modelo de negocio que le permita sobrevivir, con una comercialización publicitaria muy mermada y una creciente "canibalización" de sus contenidos por la web, sin que eso le suponga suficientes ingresos económicos. Así, las editoras españolas se debaten entre los contenidos abiertos financiados por la publicidad; los contenidos de pago, a los que se resisten los usuarios o la fórmula mixta del paywall que parece haber sido bien acogida por los lectores de, entre otros, The Times o The New York Times. En España ha probado suerte con este método El Mundo después de la experiencia frustrada de $E l$ País de años atrás.

En lo que se refiere a la radio española, afirma Legorburu Hortelano (2013: 75-134) que el empleo de las nuevas tecnologías se demoró hasta finales de los años 80, tanto en lo tocante a la producción de contenidos, como a la difusión. Después de casi siete décadas de tecnología analógica basada inicialmente en la OM y prolongada por la implantación a partir de 1957 de la FM -decisiva para su supervivencia-, se produjo una rápida modernización. El ordenador sustituyó a la máquina de escribir en la redacción y aunque en un primer momento una pléyade de nuevos dispositivos sustituyó al robusto magnetofón de cinta abierta, finalmente, también fueron desplazados por las omnipresentes terminales informáticas en red y por software específico. De nuevo, se redujo el personal técnico a favor de los periodistas, que vieron ampliadas sus tareas.

La emisora pionera en las labores de digitalización fue la Cadena SER en 1988 y la primera en ser totalmente digital fue la Cadena 40 Principales en abril de 1991. Por tanto, el proceso comenzó antes de que tuvieran lugar las primeras experiencias de emisión por DAB en 1996 y de su regulación en 1999 y de que se produjera el boom de las emisiones radiofónicas por internet en directo o a la carta.

Explica Casado Ruiz (2013: 135-182) que en España, la televisión también comenzó más tarde que la prensa su modernización tecnológica, ya que el proceso se inició en los años 80 del siglo XX y se ha prolongado hasta bien entrada la nueva centuria (2010). Han sido tres décadas de convivencia entre lo analógico y lo digital en la redacción, en los platós y en la difusión, en los que hubo incluso algunas experiencias de emisión multiplataforma como 'Sábado Cine’ y 'Sesión de noche' por Televisión Española y Radiocadena Española en los años 70 y 80 y, ya en los 90, de 'La Tertulia' por Antena 3 Radio y Antena 3 TV (Alcalá-Santaella et. al., 2012: 141).

Al igual que en los dos primeros ámbitos, en el caso de la televisión se produjo un primer punto de inflexión con la incorporación en TVE del sistema Newstar en 1989. Una vez más, de la máquina de escribir y de la cinta de vídeo, se pasó a un 
equipo informático. Fue el primer paso hacia la digitalización total en la que avanzó de nuevo TVE con su Canal 24 Horas en 1997 y que consiguió implantar de forma completa en primer lugar Tele 5 en agosto de 1998. De nuevo, el principal perjudicado desde el punto de vista laboral fue el personal técnico.

En cuanto a la difusión, se han sucedido las iniciativas de televisión de pago por satélite y cable y se ha implantado la Televisión Digital Terrestre (TDT). La primera emisora en emplear esta tecnología fue Quiero TV en 2000 -aunque no llegó a consolidarse- y todas las demás se sumaron tras la regulación legal de 2005 y el apagón analógico de abril de 2010 (Vaca Berdayes, 2013). En lo que se refiere al acceso a la imagen por internet, hoy en día se ha generalizado en todas las cadenas, aunque quizá sea TVE la que dispone de la mayor plataforma en la que, además de emisión en directo y a la carta -con sus vastos fondos documentales-, ha llevado a cabo destacadas experiencias multipantalla, como, por ejemplo, en el caso de los Juegos Olímpicos de Londres 2012 y los de Sochi 2014.

\subsection{Tres décadas de concentración empresarial}

En las anteriores líneas ha quedado patente la forma en que ha incidido en los distintos medios de comunicación el imparable proceso de digitalización, pero, ¿qué ha ocurrido desde el punto de vista empresarial en este sector? Sin duda, la segunda condición para la convergencia también se cumple. Pérez Serrano (2010: 15) afirma que "la tendencia a la concentración y a la convergencia dentro de los grupos multimedia, se hace imprescindible para comprender el estado de los medios, tanto en su vertiente empresarial como en la estructura y dinámica comunicativa".

En apenas unas décadas, en España se han sucedido las concentraciones de medios, pasando de multitud de pequeñas compañías de menor o mayor porte que operaban en prensa, radio o televisión, a cuatro grandes grupos empresariales nacionales surgidos en su mayoría de la prensa y con presencia en todos los soportes (multimedia), como explica Alcalá-Santaella (2013: 233-300).

El Grupo Vocento surgió de una editora regional del País Vasco denominada Bilbao Editorial. El éxito de sus publicaciones -El Correo y El Diario Vasco-le permitió hacerse en 1988 con una cadena de periódicos propiedad de la Conferencia Episcopal Española: Hoy, Ideal, La Verdad y Ya. Tras intentar hacer de este último periódico su cabecera nacional y no conseguirlo, años después, en 2001, pasó a controlar uno de los diarios decanos de la prensa española, $A B C$, a la vez que iba reforzándose con la compra de otros rotativos regionales consolidados. Sus ramificaciones llegan también al campo audiovisual. Es accionista de Tele 5 y de distintas productoras y obtuvo varias concesiones de frecuencias de TDT que ha alquilado a otros operadores. Asimismo, promovió en 2004 una cadena radiofónica-ABC Punto Radio-, cuyas frecuencias, una vez fracasado el proyecto en 2012, opera la Cadena COPE en régimen de alquiler.

Otro coloso de la comunicación en España es el Grupo Prisa, surgido a partir del éxito del diario El País, fundado en mayo de 1974. Su política de adquisiciones fue muy agresiva. De esta forma, la división de prensa se reforzó con la compra del 
periódico económico Cinco Días (1989) y el deportivo AS (1996) y la puesta en marcha de revistas especializadas; y se abrió al campo audiovisual en 1984 haciéndose con la mayoría del capital de la Cadena SER. Esta fue modernizada, reorganizada y completada con nuevas concesiones y con la adquisición de las frecuencias de Antena 3 Radio y de otros grupos regionales, dando lugar a una corporación radiofónica compuesta por siete cadenas: Cadena SER, Cadena 40 Principales, Cadena Dial, Ke Buena, M-80 Radio, Máxima FM y Radiolé.

Prisa también fue sumando concesiones de televisión en los sucesivos concursos públicos, lo que le permitió constituir Sogecable y poner en marcha Canal+ (de pago) y CNN+ y, más adelante, su propio canal generalista: Cuatro, en la frecuencia de Canal+; y también una red de televisiones locales -Localia TV-con la que no tuvo éxito. En lo referido a la televisión de pago por satélite, Prisa concentró sus esfuerzos en la plataforma Canal Satélite Digital, luego Digital+ y hoy Canal+/YOMVI. Hay que decir que la mayor parte de estos proyectos televisivos, bien ya no existen, bien se han transferido a otras empresas como Mediaset España o Telefónica o bien han sido transformados sustancialmente.

Unidad Editorial surgió en 2007 de dos proyectos españoles que unieron sus fuerzas y del posterior impulso de la multinacional italiana RCS Mediagroup: por un lado, la editora UNEDISA promotora del diario El Mundo, fundado en octubre de 1989 y, por otra parte, el Grupo Recoletos, propietario de los periódicos especializados Marca y Expansión e impulsor de proyecto frustrado de prensa gratuita ¿Qué? y de, entre otras revistas, las prestigiosas Actualidad Económica y Telva. Este grupo también tiene presencia en el campo audiovisual con una cadena de radio temática especializada en deportes (Radio Marca), una parte importante del capital de Unión Liberal de Radio (esRadio) y varias frecuencias de TDT que, actualmente, emplean otros operadores en régimen de alquiler después de que no obtuvieran éxito sus propias iniciativas.

El Grupo Planeta es una corporación multimedia fundada por la familia Lara en el año 1949, líder en ventas en el mundo en lengua española. En el ámbito de la comunicación, cuenta en España con un periódico nacional, La Razón, y una corporación audiovisual: Atresmedia. La Razón, fundado en noviembre de 1988 fue adquirido por Planeta poco después, aunque el grupo también tuvo presencia en la prensa gratuita con el frustrado proyecto de $A D N(2006-2011)$ y publica diversas revistas. Entre tanto, la corporación Atresmedia se ha constituido después de sucesivos cambios en la propiedad de la emisora Antena 3 TV -fundadores, Banesto-Grupo Zeta y Telefónica-y agrupa varios canales de televisión-Antena 3 TV, La Sexta, Neox, Nova y el canal de pago Gol TV-y de radio -Onda Cero, Europa FM y Onda Melodía-, fruto estas últimas de la suma de las emisoras de la Cadena Rato, Radio Amanecer, Radio España, Radio Voz y Europa FM.

RTVE es la corporación audiovisual estatal de España y tiene su origen en Radio Nacional de España (RNE), fundada en 1937, y en TVE, que empezó sus emisiones en 1956. En lo que se refiere a la radio, RNE agrupa en la actualidad seis emisoras distintas: Radio Nacional, Radio Clásica, Radio 3, Ràdio 4, Radio 5 Todo Noticias y Radio Exterior de España. Por su parte, TVE pasó de dos canales -VHF y UHF- a los siete actuales: La 1, La 2, Clan TV, Teledeporte, Canal 24 Horas, TVE HD 
y TVE Internacional. Es especialmente destacable el papel que viene desempeñando en los últimos tiempos el portal de la corporación: rtve.es.

Junto a estos grupos, hay otras corporaciones nacionales que pueden ser consideradas como multimedia. Mediaset España se centra fundamentalmente en la televisión y agrupa a Tele 5, Canal+2, Cuatro, Boing, Divinity, Energy y Factoría de Ficción. La Conferencia Episcopal Española es propietaria del Grupo COPE, corporación radiofónica propietaria de la Cadena COPE, Cadena 100, Rock FM y Megastar FM; y participa en el accionariado de 13TV. El periódico La Gaceta, la cadena televisiva Intereconomía TV -que ceso sus emisiones nacionales en febrero de 2014- y las emisoras Radio Intereconomía y Radio Inter compusieron el Grupo Intereconomía, fundado en 1995. Finalmente, el Grupo Libertad Digital tuvo su inicio en el diario electrónico del mismo nombre fundado en marzo de 2000, a partir del cual se generaron en 2007 un canal de televisión, Libertad Digital TV, que cesó sus emisiones en 2013; y en 2009, una emisora de radio: esRadio.

\subsection{La creciente polivalencia profesional del informador}

Como se ha desprende de la lectura de los epígrafes anteriores, tanto la digitalización como las sucesivas oleadas de concentración han incidido necesariamente en la formación y en las tareas en los profesionales de la información. Es innegable que, de un modo u otro, la convergencia ha afectado también a los recursos humanos, a la reorganización de las redacciones y a la aparición de los perfiles “convergentes", "polivalentes” o "flexibles”, denominaciones sin duda más razonables que las de periodista “orquesta” o "todoterreno" (Martín Bernal, 2012: 84-90).

Por una parte, la incorporación de las nuevas tecnologías ha ido simplificando los procesos productivos, haciéndolos más accesibles a los periodistas, que han ido adquiriendo un mayor protagonismo. Los informadores, antaño generadores de contenidos, son ahora también quienes les dan forma de comienzo a fin. Estas nuevas funciones han supuesto a lo largo del tiempo la desaparición de diferentes colectivos en el seno de los diferentes medios de comunicación.

Asimismo, las enormes posibilidades que han abierto las nuevas tecnologías a la elaboración de contenidos han sido un importante aliado en las operaciones de concentración empresarial, puesto que se han servido de ellas para adaptarlos automáticamente a sus diversos medios, tanto de forma horizontal, como vertical, simplificando una vez más los procesos y las necesidades de personal.

Estas nuevas posibilidades han hecho que los profesionales de la comunicación hayan ido incorporando nuevos conocimientos, adquiriendo nuevas capacidades y desarrollando nuevas destrezas, surgiendo de esta manera un profesional mucho más flexible, así como nuevos perfiles profesionales a caballo de la información y la informática, como explican Vila Fumas y Fuentes Cortina (2013: 567): 
"Esta modernización permanente, junto con los avances en los campos de convergencia e interoperabilidad, abren cada cierto tiempo nuevas posibilidades, nuevas formas de trabajar que acaban resultando en modificaciones sustanciales de los perfiles profesionales, así como en la aparición de nuevos perfiles profesionales".

Es importante reseñar que en la capacitación de los profesionales de la comunicación han intervenido tanto las empresas con sus planes de formación como la Universidad. En este sentido, hay que destacar muy especialmente el papel desempeñado por las facultades de Comunicación, que han ido adaptando los planes de estudio de sus titulaciones y el contenidos de los programas de las asignaturas para dar satisfacción a estas nuevas necesidades del mercado laboral.

\section{Metodología}

La presente investigación se desarrolló en tres fases. En lo que se refiere a la primera de ellas, el análisis estadístico -diagnóstico- de estos modelos se realizó sobre los datos recogidos mediante una metodología estadística de carácter cualitativo. De todas las técnicas de recogida de información cualitativa se seleccionó la del análisis de contenido con la finalidad de elaborar un marco teórico con el que conocer el fenómeno de la convergencia de medios en España. Este se realizó mediante el estudio de los antecedentes y la revisión de las bases teóricas y la literatura reciente. Se trataba de probar que, efectivamente, se cumplían en España las condiciones necesarias para dicho fenómeno.

De esta forma, tal y como se relata en el apartado anterior, se estudió detenidamente el recorrido histórico de la renovación tecnológica de los medios de comunicación hasta llegar al momento actual, en el que impera de forma clara la digitalización y, de forma paralela, los cambios que se han venido produciendo desde el punto de vista de los profesionales, cada vez más polivalentes y multitarea; y desde la perspectiva de la concentración empresarial.

En segundo lugar, todavía dentro de esta primera fase y una vez recogida esta información, se realizaron diferentes dinámicas de grupo con las que se obtuvo la percepción de los expertos sobre el tema a tratar. La razón de usar esta metodología se justifica en el hecho de que aporta un número considerable de información significativa sobre las variables que se quieren estudiar, así como por el hecho de que la convergencia es un fenómeno intrínsecamente cualitativo.

En concreto, se realizaron consultas con sendos paneles de expertos celebrados el día 21 de junio de 2012. En el primero participaron el Dr. Antonio Casado Ruiz (realizador de TVE), Julián de Cabo Moreno (profesor del IE Business School, director general del Consorcio para el Desarrollo Tecnológico de las PYMES y vicepresidente de la Asociación Española de Comercio Electrónico), Eladio Gutiérrez Montes (consultor audiovisual y expresidente de Impulsa TDT) y el Dr. Pere Vila Fumas (director de Tecnología, Innovación y Sistemas de RTVE). En el segundo intervinieron Miriam Dutra Schmidt (representante en España de la Corporación Multimedia Globo de Brasil), el Dr. José Alberto García Avilés (profesor de Periodismo de la Universidad Miguel Hernández de Elche e IP del Grupo GICOV), Enrique Infante 
Muñoz (director de Estrategia de DixiMedia) y el Dr. Sergio Ríos Aguilar (profesor de Telecomunicaciones de la Universidad Pontificia de Salamanca).

Con toda la información cualitativa recogida en esta primera fase (Carrizo et al, 1994) se delimitaron los procesos para la convergencia y se observó que se podían agrupar en ocho categorías: razones para la convergencia de medios, asignación de recursos para los procesos de convergencia, tipos de convergencia, realización de análisis estratégicos, sinergias de convergencia, retroalimentación; I+D, Universidad y nuevos perfiles profesionales; y publicidad y retorno de la inversión. Estas categorías daban respuesta al objetivo de obtener la diversidad de la información con respecto a las variables y también garantizaban que la información recogida fuese completa, puesto que es justamente esa diversidad la que asegura la riqueza de los resultados de la investigación.

Con la información obtenida en esta primera etapa y en el ámbito del trabajo de campo, se planificó la segunda fase de la investigación. De entre los diferentes métodos existentes (Delphi, Historias de vida, Asociación de palabras, Brainstorming, Auditoría, etc.) se escogió el de la entrevista en profundidad con cuestionario precodificado. Éste, estaba compuesto de un total de 46 preguntas tanto abiertas como cerradas, ya que de esta forma se garantizaba que las preguntas que se iban a formular, se realizasen en un orden preestablecido, recogiendo todas las variables importantes (un total de 67) y preservando la homogeneidad necesaria para poder entender la heterogeneidad de las respuestas.

En lo que se refiere a la muestra, estuvo compuesta por las principales corporaciones multimedia españolas, escogiéndose las de mayor presencia en el sector y cobertura nacional: Atresmedia, COPE, Intereconomía, Libertad Digital, Mediaset España, Prisa, RTVE, Unidad Editorial y Vocento. Las entrevistas bajo cuestionario (Nortes, 1995) a las personas a cargo de la convergencia en los grupos seleccionados se desarrollaron entre los meses de noviembre de 2012 y enero de 2013. En los casos en los que no fue posible acceder a un único responsable de la organización que fuese representativo de las ocho categorías mencionadas, la encuesta (Sierra, 2003) se realizó a los encargados de cada una o de varias de ellas, conforme al organigrama de las diferentes entidades.

En la tercera y última fase de la investigación, se elaboró un análisis estadístico (Malhotra, 2004) con la información reunida, con el fin de obtener las características de los modelos de convergencia de cada uno de los grupos multimedia objeto de estudio. La tabulación de los datos recogidos se realizó con el software SPSS (PASW Statistics 18), pudiéndose obtener la información necesaria para establecer tanto la hipótesis de trabajo, como las pautas de comportamiento dentro de los grupos multimedia y, de este modo, elaborar el análisis cualitativo y poder describir los distintos modelos de convergencia. Estos resultados se contrastaron con las tesis planteadas en el estado de la cuestión, con el fin de extraer generalizaciones significativas que contribuyesen al conocimiento de este fenómeno y obtener el grado de desarrollo en que se encuentran los diferentes grupos multimedia dentro del proceso de convergencia. 
En resumen, la metodología de la investigación, siguió los siguientes procesos: técnicas de recogida de información cualitativa a partir del análisis de contenido, la observación general y las dinámicas de grupos; técnicas de recogida de información cuantitativa a partir de la encuesta personal y, finalmente, el análisis de resultados a partir del procesamiento de los datos con SPSS y el análisis estadístico descriptivo con este mismo software.

\section{Resultados}

A continuación, se describen los resultados más relevantes obtenidos de la investigación realizada tras llevar a cabo el procesamiento de los datos obtenidos en el cuestionario, teniendo en cuenta las ocho categorías mencionadas y basándonos para su análisis en los aspectos teóricos de la convergencia previamente citados.

\subsection{Razones para la convergencia de medios}

$\mathrm{Al}$ ser consultados por las razones por las que emplean la convergencia de medios, los grupos multimedia responden haciendo referencia a cuestiones muy diversas: a la mejora o reforzamiento de su imagen de marca, a una necesidad impuesta por el mercado, a la necesidad de incrementar su audiencia y, por lo tanto de aumentar su facturación por comercialización; a la optimización en el empleo de sus recursos y, finalmente, a una mera innovación por imitación, es decir, por un simple mimetismo con el resto de empresas del sector ante el fenómeno de las tecnologías emergentes. Es el caso, por ejemplo de hacerse presentes en las redes sociales sin perseguir un objetivo concreto. Seguidamente, se analizan los resultados que se pueden consultar en el cuadro número 1, desglosados conforme a las diferentes razones anteriormente expuestas.

La imagen parece ser una razón de peso para las corporaciones a la hora de poner en práctica estrategias convergentes. No hay que olvidar que este tipo de acciones llevan aparejada cierta sensación de modernidad y de adaptación al cambio tecnológico tanto en el medio como en los propios usuarios. La mitad de los grupos consultados reconocen que esa es una de las razones por las que consideran oportuno emplear la convergencia, mientras que la otra mitad dice no tenerla en consideración.

En lo que se refiere a la optimización en el empleo de los recursos, se parte de la base de que el principal activo en cualquier medio de comunicación son los contenidos, que deben ser gestionados eficientemente a todos los niveles. Esta sí que es una razón para la convergencia en la práctica totalidad de los grupos estudiados, con solo una excepción (ver cuadro número 1).

La cuestión de la comercialización no es baladí. Las nuevas estrategias crossmedia así como la transversalidad de los contenidos, deberían ser una de las principales razones por las que realizar convergencia de contenidos, ya que también el resultado posibilita un incremento de las audiencias. Sin embargo, para grupos como Atresmedia, Intereconomía, Prisa, 
RTVE, Unidad Editorial y Vocento, el incremento de la facturación por comercialización no parece ser una de las razones por las que emplear la convergencia. En el caso de RTVE resulta comprensible, pues no se nutre de esa fuente de financiación, pero, en cambio, sí debería serlo para el resto.

La única razón que suscita unanimidad y que, por lo tanto debería condicionar buena parte de las estrategias y tácticas de los grupos con respecto a la convergencia, es que se trata de una necesidad impuesta por el mercado.

Las respuestas al respecto de la simple imitación como una de las razones para la convergencia, son negativas en prácticamente todos los casos. Sin embargo, si comparamos estas respuestas con las obtenidas en relación con la convergencia como herramienta de imagen, habría que pensar que sí que es tenida en cuenta por la mayor parte de las corporaciones multimedia a pesar de que lo nieguen o de que no sean plenamente conscientes de ello. Moreno Felices (2013: 454) arroja luz al respecto al afirmar que "el carácter cerrado y endogámico de los medios de comunicación ha producido un fenómeno de innovación por imitación del competidor”.

Cuadro 1. Razones para la convergencia
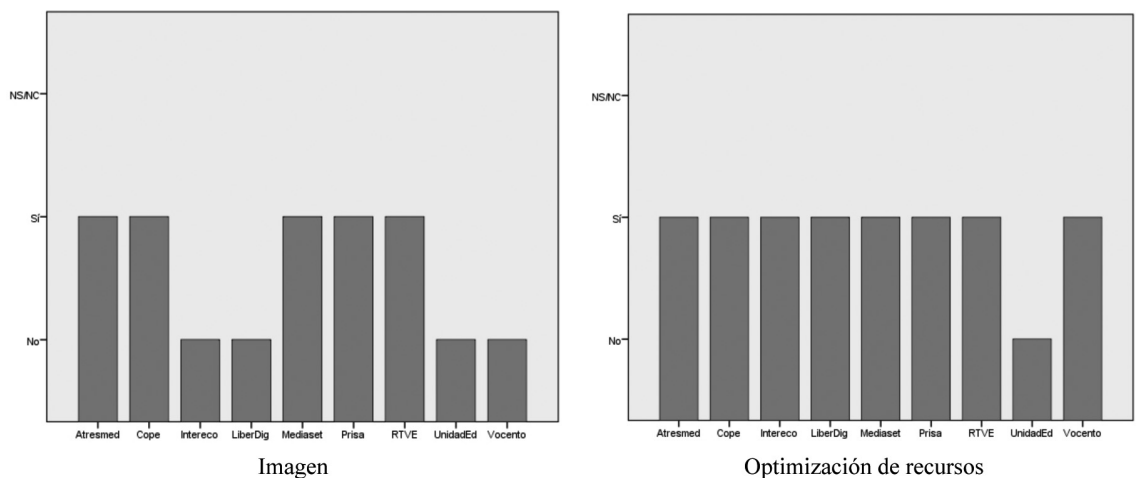

Optimización de recursos
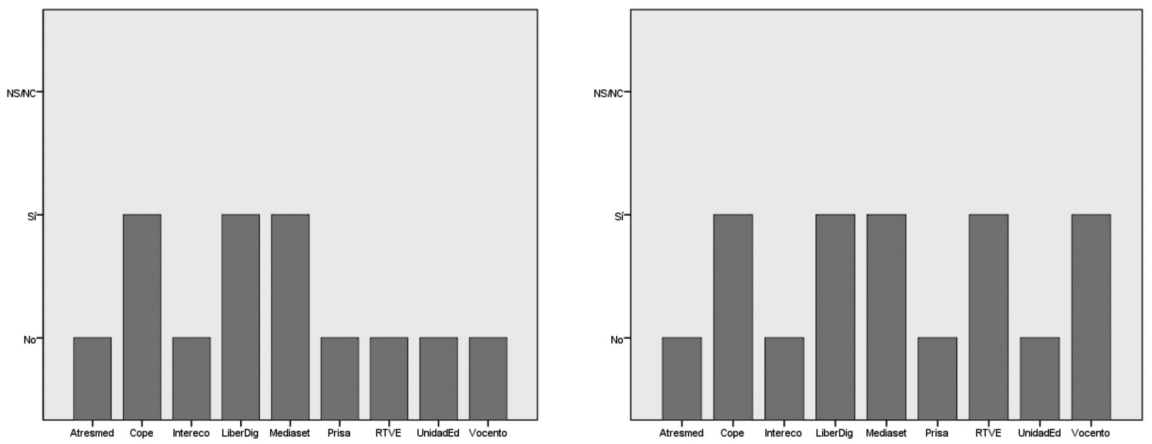

Incremento de la facturación por comercialización

Incremento de la audiencia

68 | n 19, pp. 57-83 | doxa.comunicación 

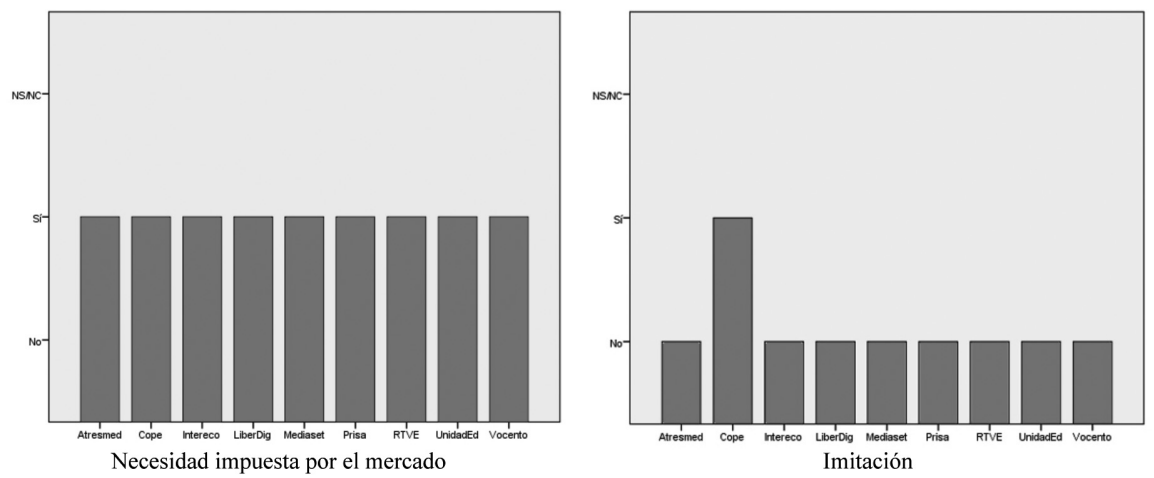

Fuente: Elaboración propia a partir de los gráficos obtenidos con SPSS

\subsection{Asignación de recursos para los procesos de convergencia}

Una de las primeras cuestiones planteadas a la hora de elaborar esta investigación era conocer si las corporaciones multimedia tenían asignado una partida económica en su presupuesto, ya sea de forma directa o indirecta, para la consecución de sus objetivos en materia de convergencia de medios.

Como se desprende de la información contenida en los cuadros 2 y 3, algunos de los grupos desconocen si es así o si una parte de los recursos económicos de que disponen se emplea con este objetivo, lo que, necesariamente, llama la atención. En cualquier caso, en términos generales, salvo excepciones, la gran mayoría destina fondos de forma directa o indirecta al desarrollo de las estrategias de convergencia.

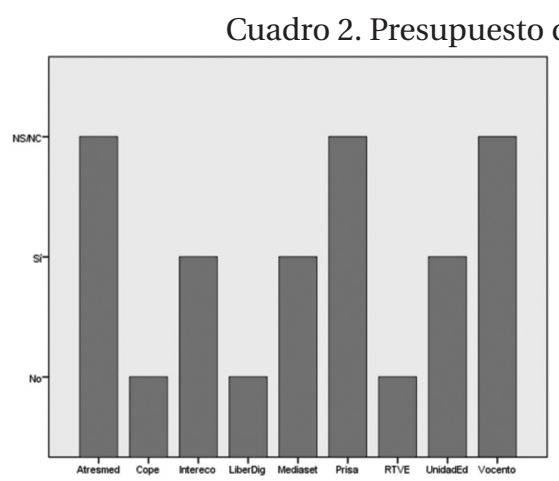

Por grupo multimedia

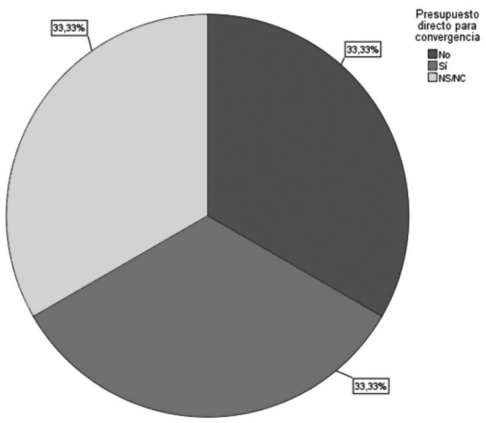

Global

Fuente: Elaboración propia a partir de los gráficos obtenidos con SPSS 
Cuadro 3. Presupuesto indirecto para convergencia
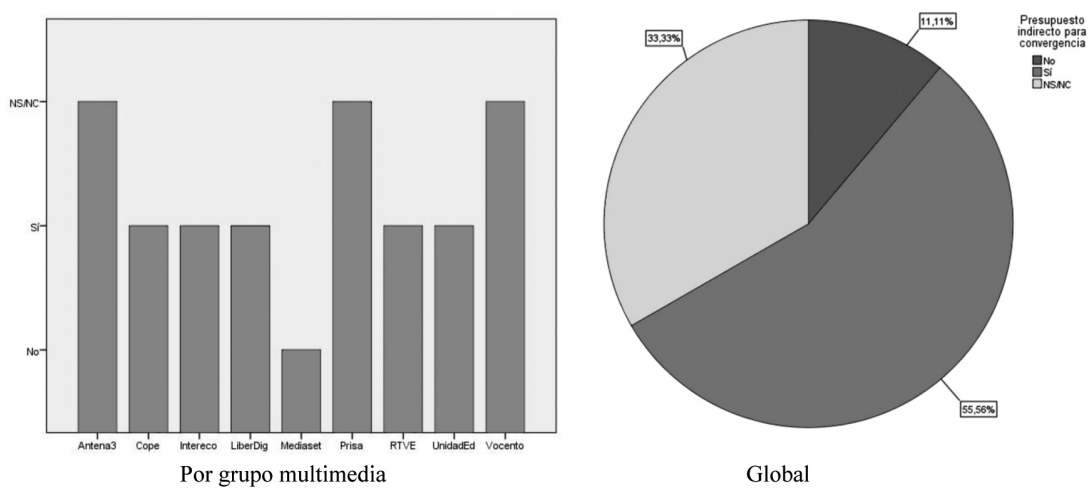

Fuente: Elaboración propia a partir de los gráficos obtenidos con SPSS

\subsection{Tipos de convergencia que realizan los grupos multimedia}

En lo que se refiere a la convergencia empresarial, parecen evidentes sus efectos en lo que se refiere a la eficacia de la gestión y así lo han detectado la mayoría de los grupos estudiados. Únicamente Mediaset España y RTVE reconocen no estar aplicándola entre sus distintas unidades de negocio.

En lo tocante a la convergencia organizacional, todos los grupos, con una única excepción (ver cuadro número 4), aseguran estan haciendo uso de la convergencia, lo que pone de manifiesto que han detectado en esta faceta una importante vía de desarrollo de la eficiencia, así como de ahorro y optimización de recursos.

Algo parecido ocurre con la convergencia tecnológica, plenamente identificada por las corporaciones por su utilidad. La tecnología digital motiva por sí misma una reconfiguración de las tareas comunicativas, abriendo nuevos horizontes para la creatividad ya que la digitalización ha contribuido a generar nuevas formas de hacer y de difundir la información. Se puede decir que el reto de la convergencia tecnológica está asumido por la mayoría de las empresas a la vista de los resultados obtenidos. 
Cuadro 4. Tipos de Convergencia
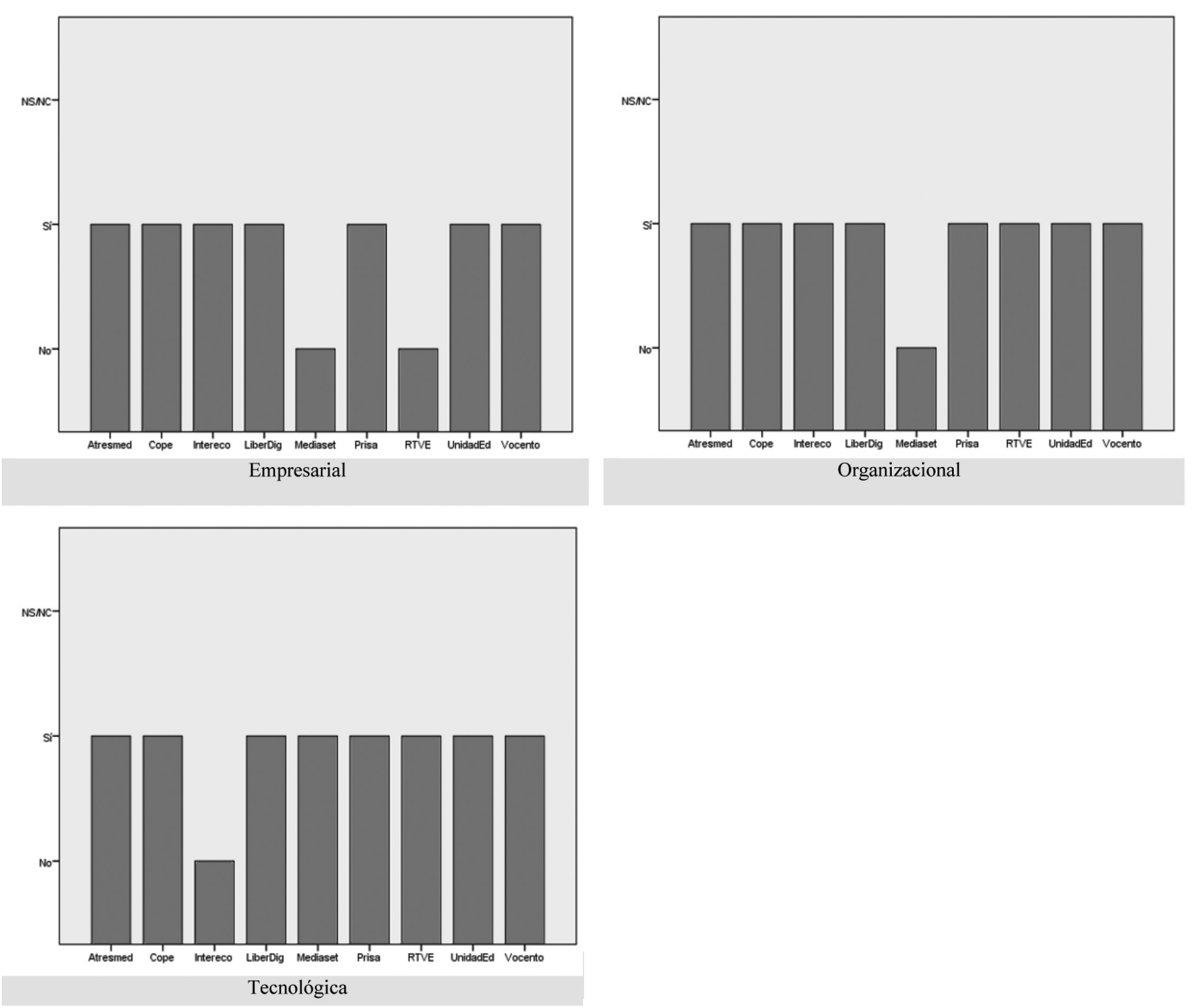

Fuente: Elaboración propia a partir de los gráficos obtenidos con SPSS

\subsection{Realización de análisis estratégicos}

En términos generales, los grupos multimedia españoles son conscientes de la importancia de la convergencia, al margen de que la empleen en mayor o menor medida o de que tengan una visión estratégica y a medio y largo plazo de su aplicación en sus diferentes procesos. En este sentido, la mayor parte de estas empresas reconocen haber llevado a cabo análisis estratégicos de debilidades y fortalezas en los últimos tiempos, como se refleja en el cuadro número 5. 
Cuadro 5. Análisis estratégico de debilidades y fortalezas

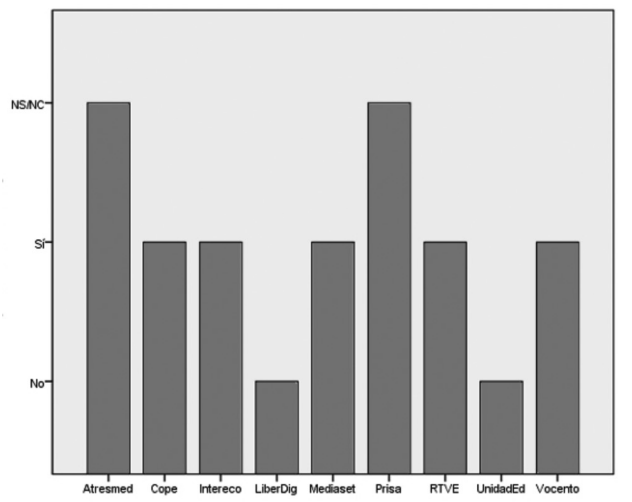

En relación con procesos de trabajo

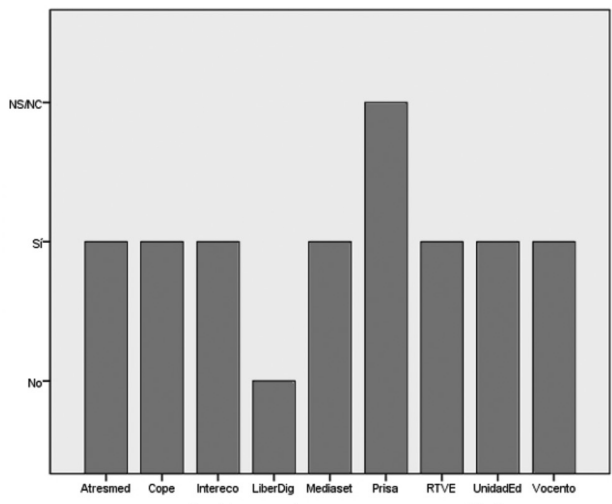

En relación con la estructura tecnológica

Fuente: Elaboración propia a partir de los gráficos obtenidos con SPSS

La inquietud por la convergencia y la preocupación por el futuro se ven claramente reflejadas en los análisis realizados con respecto a los procesos de trabajo, un aspecto este que, como se ha podido apreciar de forma reiterada, ha sido identificado por las compañías como una vía clara de desarrollo de la convergencia. La mayor parte de las corporaciones han llevado a cabo estos estudios, con algunas excepciones que afirman tajantemente que no lo han hecho.

Pero, si las corporaciones comparten un amplio consenso en lo que se refiere a los procesos de trabajo, prácticamente es unánime en lo tocante a la estructura tecnológica. Es en este aspecto donde casi todos los grupos, menos Libertad Digital, reconocen haber analizado a fondo esta cuestión para extraer todo el provecho posible de las estrategias convergentes y extenderlas en el tiempo con la necesaria previsión. En este sentido, no hay que olvidar la influencia y relación directa con en esta faceta de los perfiles profesionales más cercanos a la ingeniería y la informática.

\subsection{Sinergias de convergencia}

Para lograr la convergencia plena, resulta necesario que existan sinergias de forma simultánea en diferentes niveles y procesos, principalmente en la interconexión de las distintas redacciones donde se generan los contenidos, en la coordinación y organización de estos a todos los niveles, y desde el punto de vista tecnológico.

La observación de los resultados plasmados en el cuadro número 6 ponen de manifiesto que dos terceras partes de los grupos consultados afirman haber establecido las necesarias sinergias de comunicación y coordinación entre las redacciones de sus diferentes medios de comunicación. Llama la atención que las empresas que reconocen no tenerlas establecidas o 
suficientemente desarrolladas son corporaciones de gran tamaño tanto en número de empleados como de concentración de medios y presencia en diferentes soportes.

En cambio, el porcentaje de respuestas afirmativas es mucho mayor a la hora de confirmar que, de una u otra forma, se produce intercambio de contenidos entre las redacciones. Finalmente, es a nivel de las redes convencionales donde menos sinergias se producen, ya que no existen en prácticamente la mitad de los grupos (44,4\%). COPE, Intereconomía, Mediaset España y Prisa son los únicos grupos que aseguran haber establecido sinergias en estas tres áreas, lo que llama la atención, pues son grupos con tamaños muy dispares. Así, del estudio se desprende que la dimensión del grupo en número de empleados o su complejidad y estructura no resultan ser factores decisivos a la hora de establecer sinergias.

\section{Cuadro 6. Sinergias}

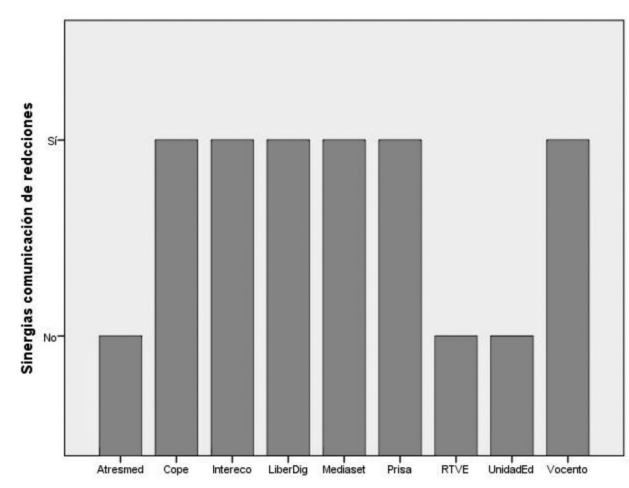

Por grupo Multimedia

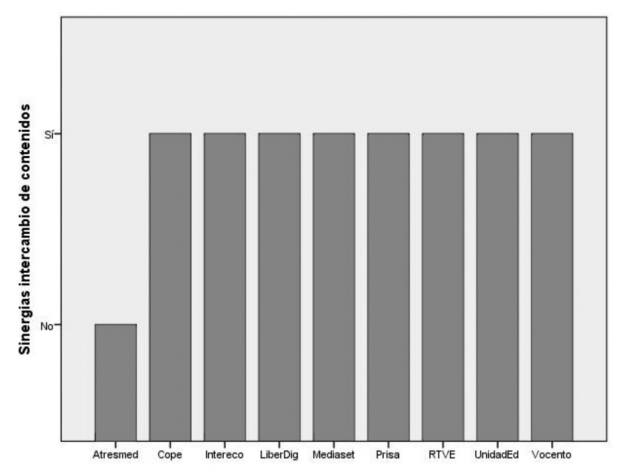

Por grupo Multimedia

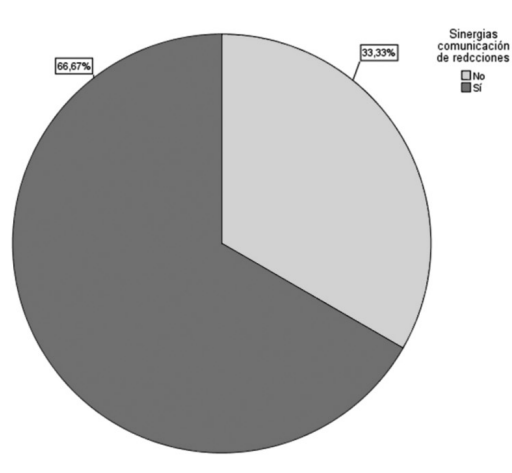

Global

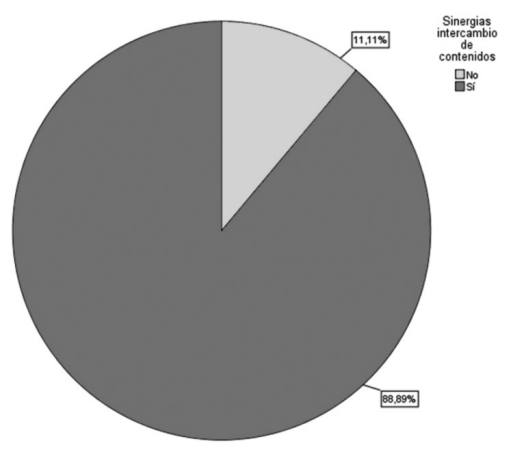

Global 


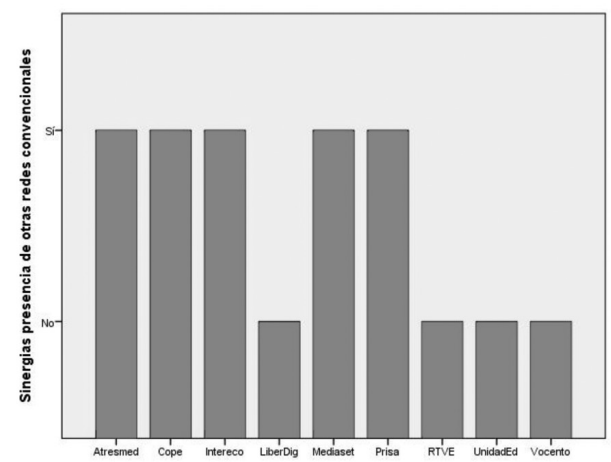

Por grupo Multimedia

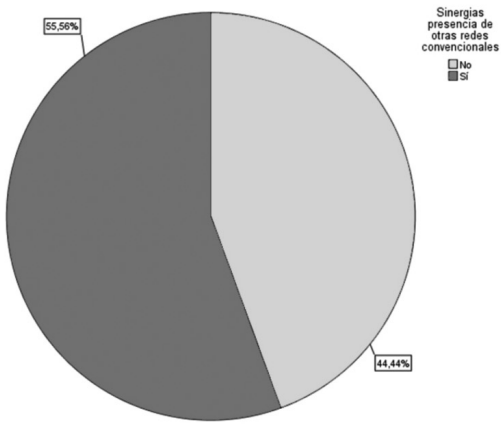

Global

Fuente: Elaboración propia a partir de los gráficos obtenidos con SPSS

\subsection{Retroalimentación}

Desde el punto de vista social, las tecnologías digitales han permitido los procesos de retroalimentación, lo que incide en un incremento de la participación de la audiencia. De este modo, los usuarios de los medios se convierten en públicos activos, en lugar de en audiencias pasivas, como ocurría con los medios analógicos (ver cuadro número 7).

A este respecto, los grupos COPE, Libertad Digital, Mediaset España, Prisa y Unidad Editorial, parecen ser especialmente conscientes de la utilidad de la retroalimentación, al entender que el incremento de la audiencia podría estar relacionado directamente con el feedback constante con sus públicos. Sin embargo, otros grupos no parecen serlo o bien desconocen si se emplea o no adecuadamente la retroalimentación, como es el caso de Atresmedia, Intereconomía o Vocento.

De igual manera, tanto el empleo de la retroalimentación de los contenidos como el porcentaje de participación de la audiencia a la hora de componer las parrillas de la programación en los medios audiovisuales debería ser una herramienta imprescindible en el objetivo estratégico de incrementar la audiencia y, en consecuencia, los ingresos por comercialización de la publicidad.

Si bien la mayor parte de los grupos consultados comparten esta línea de trabajo, no es el caso de Atresmedia, Intereconomía y RTVE. En el caso de la participación de la audiencia en el diseño de nuevos formatos y de parrillas de programación, el porcentaje de desinterés es mayor y, además, se da la paradoja de que las respuestas de algunos grupos van justamente en la línea opuesta a lo comentado anteriormente. 
Por su parte, los usuarios son cada vez más conscientes de las ventajas de la convergencia y, por lo tanto, demandan contenidos convergentes para ser consumidos en los diferentes dispositivos y vinculados a las redes sociales. Los grupos estudiados parecen estar muy sensibilizados al respecto de este fenómeno. Al menos, eso es lo que se puede deducir tanto por sus respuestas, como por la mera observación. Todos ellos dicen tener grandes aliados en estas herramientas de la web 2.0, siendo Facebook y Twitter, las más empleadas por la mayoría de los grupos.

En todo caso, a pesar del despliegue que efectúan todas las corporaciones en estas dos y en algunas otras redes sociales, lo cierto es que, como se puede apreciar, la atención que, en términos generales, prestan a sus audiencias es muy limitada y parece responder sobre todo a razones ya mencionadas anteriormente, como la imagen que proyectan en la sociedad o la simple innovación por imitación.

\section{Cuadro 7. Retroalimentación}

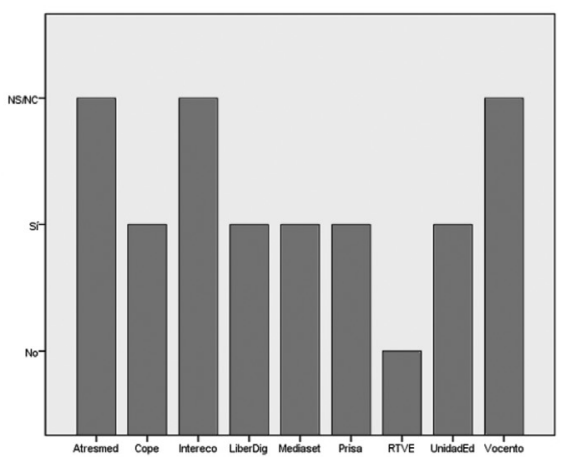

Diseño de nuevos formatos y parrillas de programación

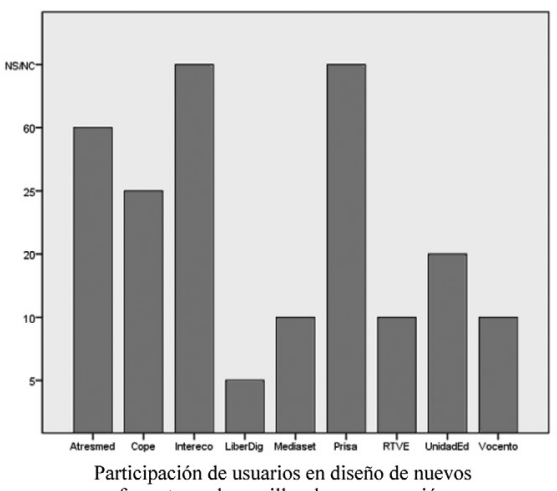

formatos y de parrillas de programación

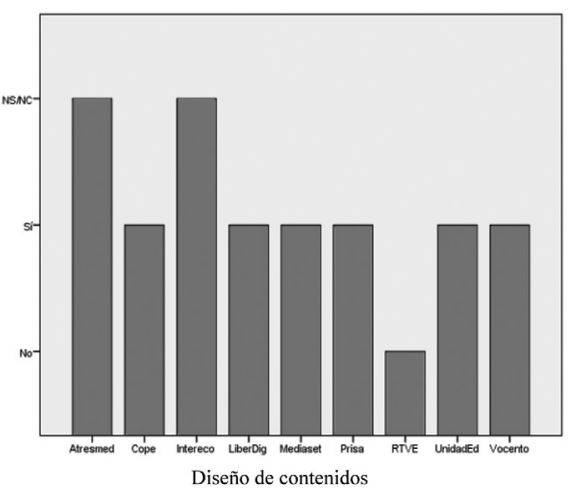

Diseño de contenidos

Fuente: Elaboración propia a partir de los gráficos obtenidos con SPSS 


\subsection{I+D, Universidad y nuevos perfiles profesionales}

La aparición de las tecnologías emergentes ha abierto un nuevo campo de investigación, no solo en materia técnica y tecnológica, sino también en el desarrollo de nuevos procesos y formatos. Por tanto, una de las cuestiones planteadas a los grupos hacía referencia a la existencia o no de un presupuesto dedicado a la investigación y el desarrollo, así como la presencia o no de colaboraciones conjuntas con la Universidad, por su capacidad como incubadora de talentos y de investigación.

De este modo, en los resultados se aprecia el hecho de que la mayoría de los grupos multimedia no dispone de un presupuesto específico para I+D, ya sea a nivel interno o externo, como es el caso de Atresmedia, COPE, Intereconomía, Libertad Digital, Prisa y RTVE. Sin embargo, estas cifras varían notablemente cuando la pregunta se extiende a las colaboraciones concretas con universidades en este sentido, puesto que al menos una parte de los grupos sí llevan a cabo proyectos específicos con las universidades o encargan la elaboración de informes y análisis a diferentes centros académicos.

Claros ejemplos de lo anteriormente expuesto son las colaboraciones que realiza Unidad Editorial con la Universidad Politécnica de Madrid, o las que viene realizando desde el año 2011 Atresmedia con las universidades Carlos III de Madrid, CEU San Pablo de Madrid, Navarra, Nebrija, Politécnica de Valencia, Pompeu Fabra de Barcelona, Pontificia de Salamanca, Rey Juan Carlos de Madrid y de Sevilla en el marco de su Proyecto de Innovación 'Mañana', iniciado en el curso académico 2010/2011.

Aún en lo que se refiere a esta cuestión, cabe reseñar la confusión que se aprecia en ocasiones en algunos de estos grupos, que no diferencian claramente los proyectos de investigación que realizan con las universidades de los convenios de cooperación educativa que tienen con ellas para las prácticas de los estudiantes.

Dentro de la batería de preguntas que hacían referencia a las nuevas rutinas y perfiles profesionales, hay que reseñar que, en la mayoría de los grupos, sí se han creado nuevos puestos relacionados con la convergencia, desempeñados por profesionales digitales que, en la mayoría de los casos, no proceden del área de la comunicación, sino que son técnicos informáticos o ingenieros.

Finalmente, en cuanto a si las corporaciones invierten o no en la formación de sus empleados en materias relacionadas con internet o los nuevos medios, es unánime la respuesta positiva, identificada claramente por los grupos multimedia como una inversión razonable y beneficiosa a corto plazo. 
Cuadro 8. Acciones formativas

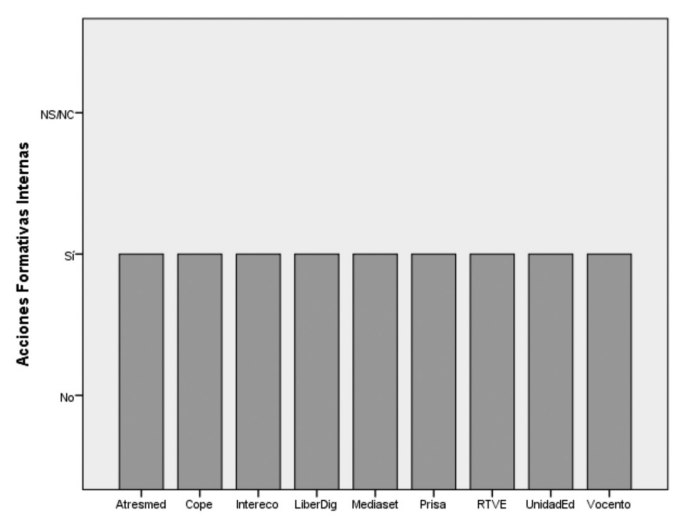

Fuente: Elaboración propia a partir de los gráficos obtenidos con SPSS

\subsection{Publicidad y retorno de la inversión}

Los usuarios, son cada vez más conscientes de las ventajas de la convergencia y, por lo tanto, demandan contenidos convergentes para ser consumidos en los diferentes dispositivos, especialmente en los móviles. A pesar de este cambio drástico en los hábitos de consumo y en los modelos de negocio y la monetización de los contenidos digitales que existen hoy en día, las corporaciones siguen patrones comunes de negocio firmemente enraizados en las décadas anteriores y basados, fundamentalmente, en la acumulación de audiencias. Y es así, a pesar de que los grupos multimedia, disponen de distintas formas de procurar el retorno de la inversión de los contenidos convergentes: mediante cobro por visión, pago por acceso o descarga, suscripciones y, por supuesto, la tradicional por comercialización de la publicidad.

En cualquier caso, resulta evidente que, en la actualidad, la publicidad no resulta suficiente para financiar los contenidos debido a la diversificación de medios y a su paulatina reducción en el tiempo. Además, las herramientas que proporcionan a los usuarios las tecnologías de la información y de la comunicación, van mucho más allá de las que emplea la publicidad actual. Es por ello, por lo que la publicidad debe aprovechar la ubicuidad de los dispositivos, las características de la digitalización y los nuevos desarrollos en el momento en el que se producen (Sánchez Martínez, 2013: 535). Al hilo de este argumento, resultaría deseable que los medios de comunicación buscasen nuevas formas para conseguir el retorno de la inversión, lejos de las tradicionales, aprovechando las estrategias crossmedia.

A pesar de todo, como se muestra en el cuadro número 9, es patente que, en la mayoría de los casos, los grupos siguen haciendo la monetización a través de la comercialización de la publicidad, quedando muy por debajo de este sistema tradicional, tanto el pago por descarga, como el pago por suscripción. 
De esta forma se puede afirmar que los resultados obtenidos en esta investigación ponen de manifiesto que a pesar del paso del tiempo aún persisten las dificultades en lo que se refiere al modelo de negocio, detectadas con anterioridad por otros estudios, como los coordinados por López García y Pereira Fariña (2010) o el desarrollado por Casero Ripollés (2012: 344).

Cuadro 9. Retorno de la inversión

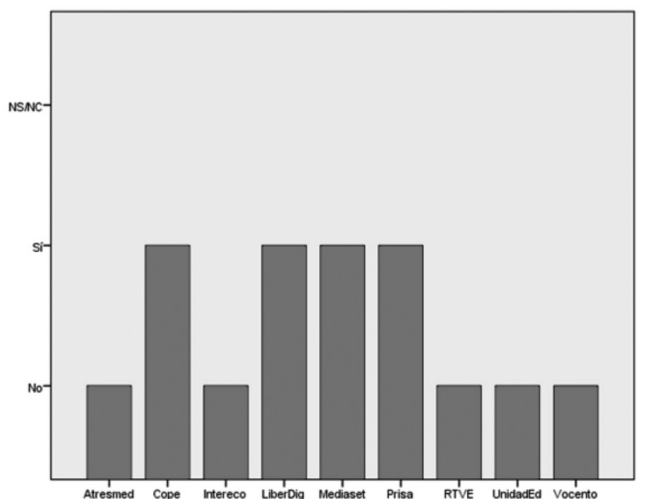

Por descarga

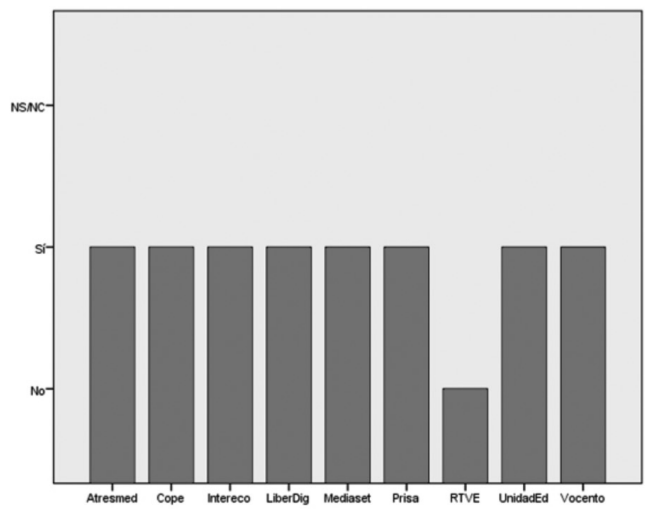

Por Publicidad

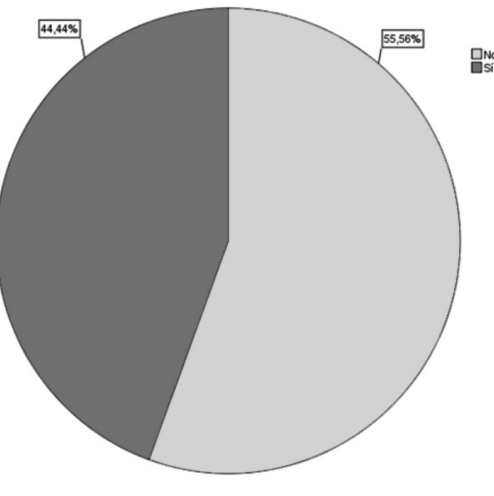

Global

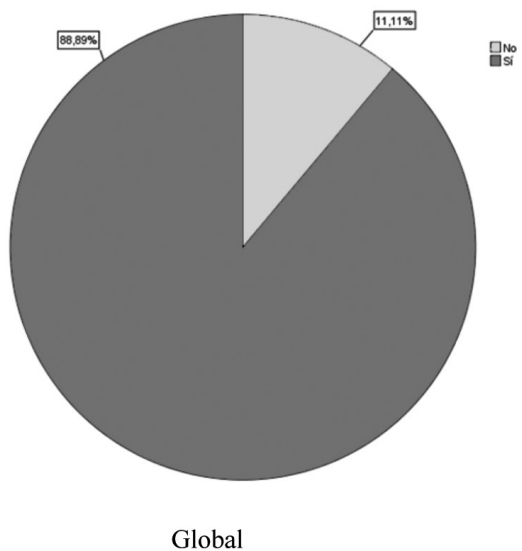




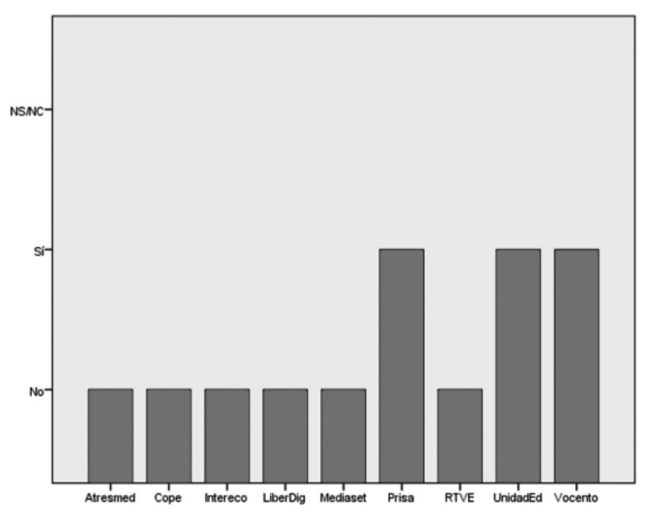

Por suscripción

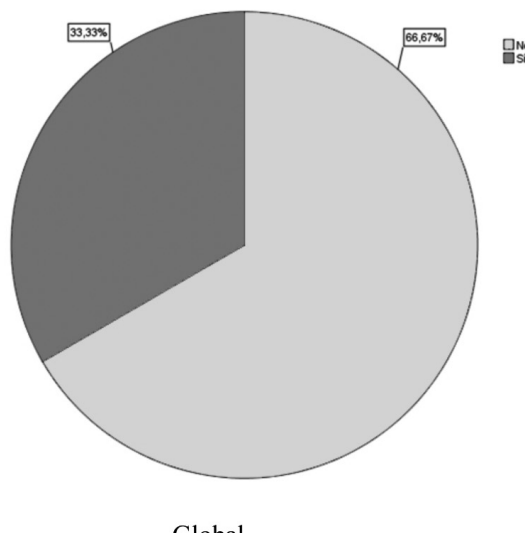

Global
品此

Fuente: Elaboración propia a partir de los gráficos obtenidos con SPSS

\section{Conclusiones}

La convergencia de medios es posible hoy en día en España gracias al desarrollo tecnológico y de internet, pero también a la concentración de medios en unos pocos grupos que se ha venido produciendo de forma sistemática en las últimas décadas. A pesar de ello, su desarrollo se encuentra en un estadio muy incipiente. Si bien la mayor parte de las corporaciones multimedia españolas son conscientes de la importancia de este fenómeno, han realizado diferentes análisis estratégicos de fortalezas y debilidades en este sentido y tienen presentes los diferentes tipos de convergencia -organizacional, empresarial, profesional y tecnológica-, lo cierto es que no acaban de identificarla como una herramienta eficaz para renovar sus modelos de negocio, adaptar a sus medios a los requerimientos de la web 2.0 y difundir sus contenidos mediante las tecnologías emergentes.

De hecho, en la mayoría de los casos estudiados la convergencia tecnológica no ha incidido de forma significativa en la creatividad y en la generación de nuevos contenidos y formatos adaptados. Esto es así, porque para la mayor parte de los grupos multimedia su aplicación se reduce al aspecto meramente económico. Al igual que ocurrió con la llegada de las nuevas tecnologías a los periódicos en el último cuarto del siglo XX, las empresas se aferran a sus modelos de negocio y las aprovechan solo como oportunidad para reducir sus costes de producción con una clara visión cortoplacista. Se podría decir que lo nuevo se adapta a lo antiguo, en vez de ocurrir al contrario.

En este sentido, la convergencia tecnológica y profesional ha supuesto una reducción muy significativa de puestos de trabajo en el entorno del personal técnico, debido a la simplificación de los procesos de producción y a la adaptación de los 
periodistas a un perfil mucho más polivalente y multitarea. Así, los profesionales de la información asumen nuevas tareas y adoptan nuevos perfiles, aunque también se detecta la incorporación de técnicos -fundamentalmente, expertos en informática- que desempeñan labores relevantes en los diferentes procesos productivos. Esta sí es una faceta de la convergencia claramente reconocida y valorada por las empresas, en la que no dudan en invertir a través de becas, cursos de formación, etc., pues obtienen un beneficio económico inmediato.

En cambio, de la investigación se desprende la existencia de serias dificultades organizativas en los grupos para generar sinergias de convergencia y cambio entre sus distintos medios. Este fenómeno es apreciable tanto en las grandes corporaciones, como en las empresas de tamaño más reducido. Claro ejemplo de ello es bien la falta de redacciones conjuntas en los grupos, bien la falta de una auténtica convergencia redaccional. Las rutinas establecidas a lo largo del tiempo se resisten al cambio y, por el momento, parecen ganar la partida.

En lo que se refiere a las estrategias de comercialización, éstas siguen siendo autónomas. El crossmedia, continúa siendo un concepto esquivo y el retorno de la inversión se sigue haciendo, fundamentalmente, a través de la comercialización de la publicidad, lo que a medio plazo se plantea como un grave problema si se tiene en cuenta, por una parte, su reducción sostenida en el tiempo y, por otro lado, las muchas formas que tiene en su mano el usuario para esquivarla, sobre todo en los nuevos soportes. Sí es cierto que, aunque tímidamente, el pago por descarga se está convirtiendo en una opción plausible para los grupos multimedia, un fenómeno que se podría ver favorecido por la aparición del concepto de micropago.

El incremento de la audiencia siempre ha sido uno de las necesidades fundamentales de los medios, principalmente porque a mayor audiencia se presume un aumento de los ingresos por comercialización publicitaria. Sin embargo, la atención que le prestan los medios a su audiencia sigue dejando mucho que desear. Una vez más, las empresas se dejan llevar por la inercia y si bien aparentan buscar la interacción con sus usuarios, lo cierto es que son contados los casos en que verdaderamente les tienen en cuenta para diseñar sus formatos y sus parrillas. A menudo, se trata de una mera cuestión de imagen o de mimetización con un entorno en el que las redes sociales están cada vez más presentes.

Finalmente, resulta fundamental para el avance de la convergencia de medios en España que exista una mayor conciencia en las corporaciones de la necesidad de promover la investigación a medio y largo plazo. El reducido porcentaje de inversión en I+D, así como la falta de colaboración con el mundo académico a la hora de desarrollar estudios específicos, pone de manifiesto la poca importancia que se le otorga. Sin duda, se trata de un error estratégico de primer orden.

\section{Referencias bibliográficas}

Alcalá-Santaella, M. (2013): “Las editoriales y los grandes periódicos, origen de los grupos multimedia”, en Legorburu Hortelano, J. M. (coord.): Modelos de convergencia de medios en España I: concentración, digitalización y nuevos soportes. Madrid: CEU Ediciones, pp. 233-300.

$80 \mid n^{\circ}$ 19, pp. 57-83 | doxa.comunicación 
Alcalá-Santaella, M.; Alcudia Borreguero, M.; Legorburu Hortelano, J. M. (2012): "La participación de la audiencia en la convergencia radio-televisión. El caso de las tertulias de análisis político”, en Berrocal Gonzalo, S.; Campos Domínguez, E. (coords.): La investigación en periodismo político en el entorno de los nuevos medios. Salamanca: SEP, pp. $139-158$.

Carrizo Sainero, G.; Irureta-Goyena Sánchez, P.; López de Quintana Sáenz, E. (1994): Manual de fuentes de información. Madrid: CEGAL.

Casado Ruiz, A. (2013): “La digitalización total: la nueva televisión”, en Legorburu Hortelano, J. M. (coord.): Modelos de convergencia de medios en España I: concentración, digitalización y nuevos soportes. Madrid: CEU Ediciones, pp. 135-182.

Casero-Ripollés, A. (2012): “Contenidos periodísticos y nuevos modelos de negocio: evaluación de servicios digitales”, El profesional de la información, v. 21, n. 4, pp. 341-346.

Castells Oliván, M. (2001): La Galaxia Internet. Reflexiones sobre Internet, empresa y sociedad. Madrid: Areté.

Dailey, L. ; Demo, L. ; Spillmann, M. (2005): “The Convergence Continuum: A Model for Studying Collaboration Between Media Newsrooms”, Atlantic Journal of Communication, v. 13, n. 3, pp. 150-168.

De Sola Pool, I. (1983): Technologies of Freedom. Cambridge: Harvard University Press.

Dennis, E. E. (2003): “Prospects for a Big Idea, is there a future for convergence?” International Journal of Media Management, n. 5, pp. 7-11.

Dennis, E.; Pavlik, J. V. (1993): “The coming for convergence and its consequences”, en Pavlik, J. V.; Dennis, E. E. (eds.): Demystifying Media Technology. Mountain View: Mayfield Publishing, pp. 1-3.

Diezhandino Nieto, P. (coord.) (2012): El periodista en la encrucijada. Madrid: Fundación Telefónica y Ariel.

Fidler, R. (1997): Mediamorphosis. Understanding New Media. Thousand Oaks: Pine Forge Press.

Flynn, B. (2000): Digital TV, Internet \& Mobile Convergence Developments and projections for Europe. Londres: Phillips Global Media.

García Avilés, J. A. (2013): “El desarrollo de los medios en España: convergencia e innovación”, en Legorburu Hortelano, J. M. (coord.): Modelos de convergencia de medios en España I: concentración, digitalización y nuevos soportes. Madrid: CEU Ediciones, pp. 183-230.

González Díez, L.; Pérez Cuadrado, P. (2013): “De la digitalización a la convergencia: los cambios en la producción informativa sobre los modelos de negocio de la prensa diaria española”, en Legorburu Hortelano, J. M. (coord.): Modelos de convergencia de medios en España I: concentración, digitalización y nuevos soportes. Madrid: CEU Ediciones, pp. 25-74.

Gordon, R. (2003): “The meanings and implications of convergence”, en Kawamoto, K. (ed.): Digital Journalism: Emerging Media and the Changing Horizons of Journalism, Nueva York/Oxford: Rowman \& Litteflied Publishers, pp. 57-73.

Jenkins, H. (2004): “The Cultural Logics of Media Convergence”, International Journal of Cultural studies, n. 7, pp. 33-43. 
Jenkins, H. (2006): Convergence Culture. Where Old and New Media Collide. Nueva York: New York University Press.

Legorburu Hortelano, J. M. (2013): “La digitalización de la radio. Un nuevo concepto en la producción y difusión de contenidos”, en Legorburu Hortelano, J. M. (coord.): Modelos de convergencia de medios en España I: concentración, digitalización y nuevos soportes. Madrid: CEU Ediciones, pp. 75-134.

López García, X.; Pereira Fariña, X. (coords.) (2010): Convergencia digital. Reconfiguración de los medios de comunicación en España. Santiago de Compostela: USC.

Malhotra, N. K. (2004): Investigación de mercados. México: Pearson Educación.

Martín Bernal, O. (2012): "Retratos robot del periodista en el entorno digital. Los nuevos perfiles profesionales y laborales" en Diezhandino Nieto, P. (coord.): El periodista en la encrucijada. Madrid: Fundación Telefónica, Ariel, pp. 79-112.

Moreno Felices, P. (2013): “Convergencia estructural en medios: un delicado equilibrio entre personas, procesos y tecnología”, en Legorburu Hortelano, J. M. (coord.): Modelos de convergencia de medios en España I: concentración, digitalización y nuevos soportes. Madrid: CEU Ediciones, pp. 451-512.

Negroponte, N. (1995): El mundo digital. Barcelona: Ediciones B.

Nortes Checa, A. (1995): Encuestas y precios. Madrid: Síntesis.

Pavlik, J. V. (2004): "A Sea-Change in Journalism: Convergence, Journalists, their Audiences and Sources", Convergence, n. 10, pp. 21-29.

Pérez Serrano, M. J. (2010): “Un método para analizar los procesos de concentración mediática”, Doxa Comunicación, n. 11, pp. 13-29.

Salaverría Aliaga, R. (2003): ¿ Hacia dónde se dirige la convergencia de medios?. Navarra: Mediacción Consultores, Universidad de Navarra. http://dspace.unav.es/dspace/bitstream/10171/5107/1/medoc-0411-01.pdf

Salaverría Aliaga, R..; García Avilés, J. A.; Masip Masip, P. (2008): "Media Convergence as a Research Concept: A Proposal for its Theoretical and Operational Definition”, II European Communication Conference, Barcelona: ECREA.

Sánchez Martínez, M. (2013): “Nuevos procesos de la publicidad digital: Crossmedia, Transmedia y Apps”, en Legorburu Hortelano, J. M. (coord.): Modelos de convergencia de medios en España I. Digitalización, concentración y nuevos soportes. Madrid: CEU Ediciones, pp. 509-540.

Sierra Bravo, R. (1997): Técnicas de Investigación Social. Madrid: Thomson.

Vaca Berdayes, R. (2013): "Los operadores televisivos en España. De la televisión única a las concentraciones de la TDT” en Legorburu Hortelano, J. M. (coord.): Modelos de convergencia de medios en España I: concentración, digitalización y nuevos soportes. Madrid: CEU Ediciones, pp. 369-450. 
Vila Fumas, P.; Fuentes Cortina, G. (2013): "La convergencia en los nuevos soportes y en las redes sociales. Cómo la tecnología facilita la convergencia”, en Legorburu Hortelano, J. M. (coord.): Modelos de convergencia de medios en España I: concentración, digitalización y nuevos soportes. Madrid: CEU Ediciones, pp. 541-593. 